\title{
Estudo dos aspectos alimentares de Trichiurus lepturus (Pisces: Perciformes) na costa Maranhense, Brasil
}

\author{
Study of feeding aspects of Trichiurus lepturus (Pisces: Perciformes) on the coast of Maranhense, \\ Brazil \\ Estudio de aspectos alimentarios de Trichiurus lepturus (Piscis: Perciformes) en la costa de \\ Maranhense, Brasil
}

\author{
Alice Viene Serra Garcia \\ ORCID: https://orcid.org/0000-0001-5111-3425 \\ Universidade Estadual do Maranhão, Brasil \\ E-mail: aliceeviene16@gmail.com \\ Rosiele Assunção Matão \\ ORCID: https://orcid.org/0000-0002-0505-6738 \\ Universidade Estadual do Maranhão, Brasil \\ E-mail: rosieleassuncao@gmail.com \\ Yago Bruno Silveira Nunes \\ ORCID: https://orcid.org/0000-0002-0890-1730 \\ Universidade Federal Rural da Amazônia, Brasil \\ E-mail: ybruno3098@gmail.com \\ Jailza Freitas \\ ORCID: https://orcid.org/0000-0003-2911-3597 \\ Universidade Estadual do Maranhão, Brasil \\ E-mail: jailza.freitas777@gmail.com \\ Jackellynne Fernanda Farias Fernandes \\ ORCID: https://orcid.org/ 0000-0002-6559-9240 \\ Universidade Federal de Santa Catarina, Brasil \\ E-mail: jackellynnefffernandes@gmail.com \\ Marina Bezerra Figueiredo \\ ORCID: https://orcid.org/0000-0001-7485-8593 \\ Universidade Estadual do Maranhão, Brasil \\ E-mail: marina_fig@hotmail.com
}

\begin{abstract}
Resumo
O trabalho consiste em conhecer os aspectos da dieta natural do Trichiurus lepturus, uma vez que o mesmo apresenta grande valor comercial para o estado do Maranhão. A espécie analisada foi proveniente da pesca comercial, cuja captura aconteceu no município da Raposa - MA, entre os períodos de janeiro a dezembro de 2019. Foram estudados 144 organismos. Para cada espécie de presa, calcularam-se as frequências de ocorrência numérica e de biomassa e Índice de Importância Relativa (IIR) na dieta. Quanto aos itens alimentares presentes na dieta da espécie, observou-se uma dieta baseada em peixes, IND, sardinha (Sardinella brasiliensis) e camarões peneídeos. A região em estudo pode ser caracterizada como local de alimentação da espécie, visto que apresenta dieta composta por organismos neríticos de hábitos pelágicos associados a áreas costeiras e estuarinas, sendo peixes e IND os itens mais específicos na dieta. Observou-se que durante a estação seca (julho a dezembro), quando a produtividade biológica é baixa, a atividade alimentar aumenta. A dieta do macho é baseada em resto de peixes, enquanto a IND da fêmea é dominante. Todos os exemplares imaturos machos apresentaram seus estômagos totalmente preenchidos e as fêmeas apresentaram uma grande quantidade de itens não identificados (IND).
\end{abstract}

Palavras-chave: Hábitos alimentares; Conteúdo estomacal; Peixe.

\begin{abstract}
The work consists of knowing the aspects of the natural diet of Trichiurus lepturus since it has great commercial value for the state of Maranhão. The species analyzed came from commercial fishing, which was captured in the municipality of Raposa - MA, between January and December 2019. A total of 144 organisms were studied. The frequencies of numerical and biomass occurrence and Relative Importance Index (RII) in the diet were calculated for each prey species. As for the food items present in the species' diet, a diet was observed based on fish, unidentified items, sardines (Sardinella brasiliensis), and penaeid shrimp. The region under study can be characterized as a feeding place for the species. It has a diet composed of neritic organisms with pelagic habits associated with coastal
\end{abstract}


and estuarine areas, fish item being the most specific items in the diet. It was observed that during the dry season (July to December), when biological productivity is low, feeding activity increases. The male's diet is based on fish rest, while the female's unidentified items is dominant. All immature males had their stomachs filled, and females had many unidentified items.

Keywords: Eating habits; Stomach contents; Fish.

\section{Resumen}

El trabajo consiste en conocer los aspectos de la dieta natural de Trichiurus lepturus, ya que tiene un gran valor comercial para el estado de Maranhão. Las especies analizadas provienen de la pesca comercial, la cual fue capturada en el municipio de Raposa - MA, entre enero y diciembre de 2019. Se estudiaron un total de 144 organismos. Para cada especie de presa, se calcularon las frecuencias de ocurrencia numérica y de biomasa y el Índice de Importancia Relativa (RII) en la dieta. En cuanto a los alimentos presentes en la dieta de la especie, se observó una dieta a base de pescado, elementos no identificados, sardinas (Sardinella brasiliensis) y camarones peneidos. La región en estudio se puede caracterizar como un lugar de alimentación de la especie, ya que tiene una dieta compuesta por organismos neríticos con hábitos pelágicos asociados a áreas costeras y estuarinas, siendo los peces los ítems más específicos de la dieta. Se observó que durante la época seca (julio a diciembre), cuando la productividad biológica es baja, aumenta la actividad alimentaria. La dieta del macho se basa en el reposo de los peces, mientras que la IND de la hembra es dominante. Todos los machos inmaduros tenían el estómago completamente lleno y las hembras tenían una gran cantidad de elementos no identificados (ENI).

Palabras clave: Hábitos alimentarios; Contenido del estómago; Pescado.

\section{Introdução}

A atividade extrativista, sobretudo a pesca, é uma das ocupações mais antigas e importantes desenvolvidas pelo homem para a manutenção e fixação das civilizações nas zonas costeiras (Cardoso et al., 2008). Para tanto, alguns aperfeiçoamentos tecnológicos foram decisivos para evolução da pesca ao crescimento populacional (Almeida, 2009) como, melhor autonomia das embarcações e capacidade de suporte, o que possibilitou um aumento na extração dos estoques, distribuição e comercialização do pescado (Fonteles-Filho, 2011).A região Nordeste é composta por nove estados sendo o Maranhão, o que possui o segundo maior litoral do Brasil, com $640 \mathrm{~km}$ de linha de costa, em que a economia é basicamente proveniente da produção de pescado pela pesca artesanal (Almeida, 2009). Nunes e Piorski (2011), informam que os recursos pesqueiros da zona costeira maranhense são dominantes e conduzidos tanto em águas estuarinas quanto marinhas, e que há uma existência e dependência de uma alta biomassa e elevada produtividade na região.

O Trichiurus lepturus é uma espécie cosmopolita denominada comumente de peixe-espada, e na região maranhense é conhecido como guaravira. Distribui-se em águas quentes e temperadas, entre $60^{\circ} \mathrm{N}$ e $45^{\circ} \mathrm{S}$, com salinidades entre 33 e $36 \mathrm{ppm}$ e temperaturas superiores à $16^{\circ} \mathrm{C}$ (Magro, 2006). No Oceano Atlântico, a espécie se distribui do Canadá ( 40 $\left.\mathrm{N}\right)$, até a Argentina $\left(\sim 37^{\circ} \mathrm{S}\right)$, desde a linha de costa até profundidades em torno de 350m (Martins \& Haimovici, 2000; FAO, 2005). Segundo Chiou et al. (2006) e Bittar (2008), essa espécie apresenta hábito demerso-pelágico, sendo considerado oportunista e voraz quanto à alimentação.

O conhecimento sobre a alimentação é uma importante ferramenta para se obter amostras sobre as populações aquáticas e o funcionamento da troca de energia local, entretanto, é necessário compreender o processo de funcionamento do ambiente, os aspectos ecológicos e biológicos, com a finalidade de promover medidas de manejo para conservação e manutenção dos estoques pesqueiros comercialmente importantes (Coelho et al. 2010). De acordo com Andrade et al. (2011), a dieta alimentar natural quando é autóctone se mostra eficiente para adquirir medidas de manejo que visem o controle do uso dos estoques de organismos aquáticos, com a elucidação das variações nas perspectivas biológicas das espécies.

Para compreender a relação potencial ecológica do T. lepturus é necessário um melhor conhecimento de seus hábitos alimentares. Assim, o trabalho objetiva obter informações sobre a dieta da referida espécie, uma vez que os resultados servirão para fornecer bases biológicas para o uso sustentável e uma posterior gestão na zona costeira do Maranhão. 


\section{Metodologia}

\section{1 Área de Estudo}

O município de Raposa está localizado a $30 \mathrm{~km}$ de São Luís (encontra-se entre as coordenadas de $02^{\circ} 25^{\prime}$ ' 22'”S e $44^{\circ}$ 05’ 21'W), capital do Estado do Maranhão, e possui uma população de 29.167 habitantes (IBGE, 2012), que se distribuem em uma superfície de cerca de $66 \mathrm{Km}^{2}$. É limitado ao norte pelo Oceano Atlântico; ao sul pelos municípios de Paço do Lumiar e de São José de Ribamar; a leste pela ilha de Cururupu e a baia de São Marcos e a oeste pelo município de São Luís (Figura 1). O clima local é bem distinto e dividido em dois períodos sazonais, o período chuvoso, compreende os meses de janeiro a junho, enquanto a época da estiagem equivale aos meses de julho a dezembro (Azevedo et al. 2008).

Figura 1 - Localização do município de Raposa Estado do Maranhão, Brasil.

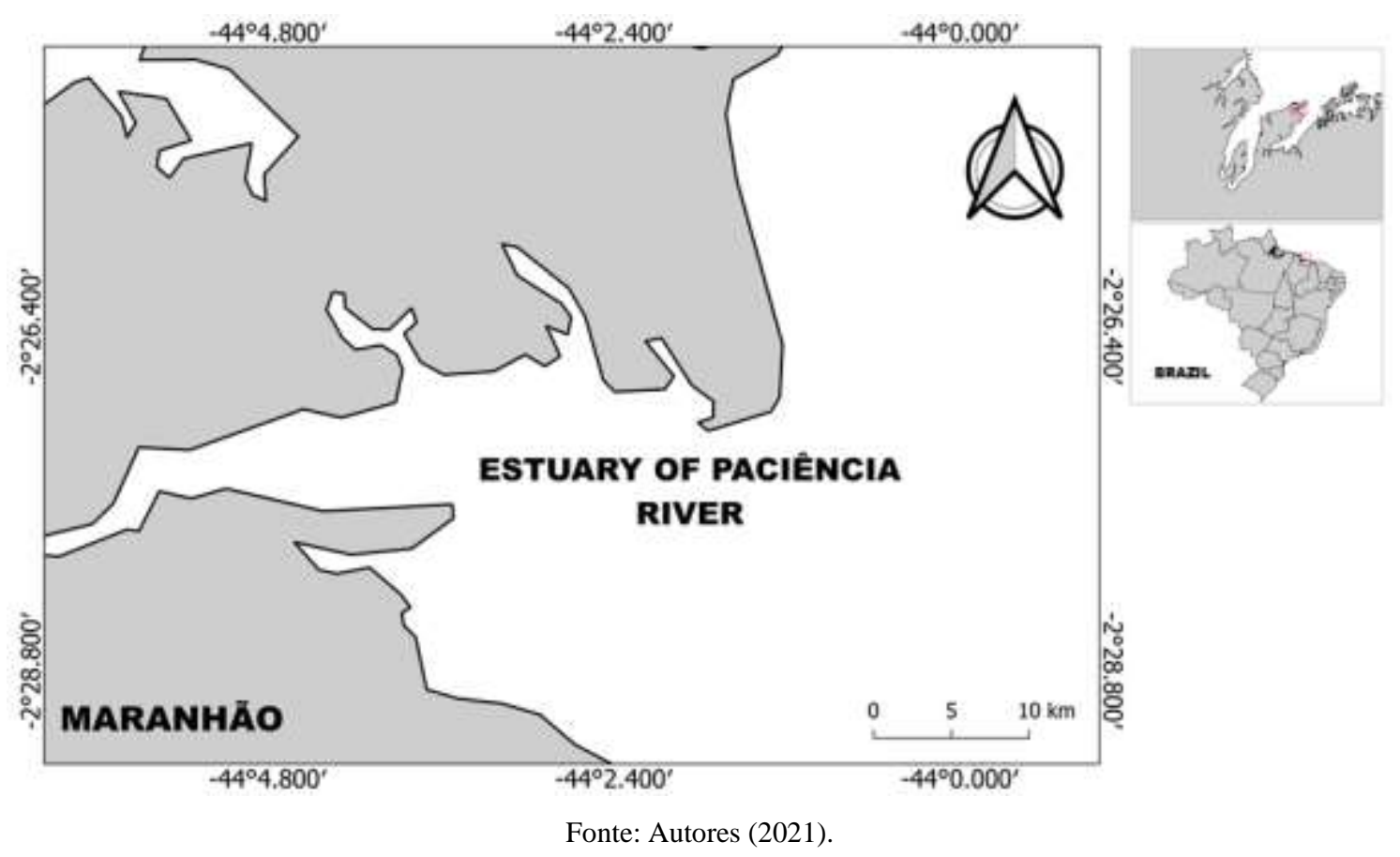

\subsection{Procedimentos Metodológicos}

Um total de 144 organismos foram obtidos no município da Raposa - MA através da pesca comercial, as coletas ocorreram os meses de janeiro a dezembro de 2019. Os exemplares foram levados ao Laboratório de Biologia Pesqueira (BIOPESQ) da Universidade Estadual do Maranhão (UEMA), onde foi realizado o processamento do material biológico. Os espécimes foram seccionados longitudinalmente na sua parte ventral com o auxílio de tesoura e pinça para a remoção dos estômagos, em seguida, foram abertos e os itens identificados ao menor táxon possível, uma vez coletados os conteúdos estomacais foram, medidos e pesados.

Para cada item encontrado foram obtidos os dados: número e peso úmido $(\mathrm{g})$ de cada presa individual e grau de digestão das presas. Com relação ao grau de digestão foi determinado três classificações: digerido, não digerido ou semidigerido. A limitação do período alimentar foi estruturada pela identificação do grau de repleção estomacal dos exemplares através de uma avaliação visual do grau de preenchimento dos estômagos. Para a pesquisa também foi adicionado e utilizado padrões de diferenciação gonadais para identificação dos sexos e uma escala maturacional para distinguir juvenis dos adultos conforme Vazzoler (1996).

Os dados pluviométricos acumulados de janeiro a dezembro de 2019 foram obtidos no banco de dados do Instituto Nacional de Meteorologia - INMET (http://www.inmet.gov.br) para cada mês de coleta. 


\subsection{Tratamento numérico e estatístico}

A estratégia alimentar de T. lepturus foi analisada pelo método de Hyslop (1980). Através deste método, as informações sobre ecologia alimentar de predadores são obtidas pela relação sua frequência de ocorrência (\%Fo), calculados da seguinte forma: $\mathrm{FO}=(\mathrm{Ni} \times 100 / \mathrm{N})$, onde; $\mathrm{FO}=$ frequência de ocorrência do item i na dieta da espécie; Fni= número de estômagos contendo a presa i; $\mathrm{N}$ = número total de estômagos com alimentos na amostra. Da mesma forma foi calculada a abundância Relativa $\mathrm{N}=(\Sigma \mathrm{Ni} / \Sigma \mathrm{NTi}) \times 100$, onde $\mathrm{Ni}=$ número de indivíduos de itens de presa $\mathrm{i} ; \mathrm{NTi}=$ número total de todos os itens de presas e peso percentual dos itens $\mathrm{Pi}=\mathrm{Pi} / \mathrm{Pt} \times 100$ no qual o $\mathrm{Pi}=$ peso das presas $\mathrm{i} ; \mathrm{Pt}=$ peso total de todas as presas; e o índice de importância relativa (IIR) (Pinkas et al., 1971) usando a formula: IIR $=(\% \mathrm{Ni}+\% \mathrm{Pi}) * \% \mathrm{FO}$, onde $\% \mathrm{Ni}$, \%Pi e \%FO são a abundância relativa, biomassa e frequência de ocorrência, de modo respectivo.

A diversidade dos itens foi calculada através do índice de Shannon-Wiener (1963). A riqueza de espécies foi proposta através do índice de Margalef (1958) e o índice de Equabilidade de Pielou (1966) usado para identificar a distribuição dos itens. A matriz de similaridade foi baseada na distância Bray-Curtis para o agrupamento dos itens alimentares. Por fim, o teste $t$ foi aplicado para verificar se houve variação significativa $(p<0.05)$ entre os períodos sazonais (chuvoso e estiagem). As análises estatísticas foram executadas nos programas Past 3.14.

\section{Resultados}

A caracterização dos estômagos do T. lepturus mostrou que no período chuvoso o comprimento dos estômagos desses organismos apresentava um tamanho significativamente maior e um volume menor, se comparado ao período de estiagem, ou seja, a relação comprimento e volume demonstrou ser inversamente proporcional (Tabela 1).

Tabela 1 - Caracterização dos estômagos analisados do Trichiurus lepturus capturados na costa maranhense.

\begin{tabular}{|c|c|c|c|c|c|c|c|c|}
\hline \multirow{2}{*}{$\begin{array}{c}\text { Períodos } \\
\text { estacionários }\end{array}$} & \multicolumn{3}{|c|}{ Comprimento dos estômagos $(\mathrm{cm})$} & \multicolumn{5}{|c|}{ Peso dos estômagos (g) } \\
\hline & Min & Max & Média & & Min & Max & Média & \\
\hline Chuvoso & 8 & 35 & $17,92 \pm 5,6$ & & 1,48 & 39,14 & $8,73 \pm 8,86$ & \\
\hline Estiagem & 8 & 28 & $15,98 \pm 3,86$ & $\mathrm{p}<0,05$ & 1,23 & 78,28 & $12,97 \pm 17,97$ & $\mathrm{p}<0,05$ \\
\hline
\end{tabular}

Nota: Chuvoso igual aos meses de janeiro a julho; Estiagem igual aos meses de agosto a dezembro. Fonte: Autores (2021).

Foi identificado uma variedade de itens alimentares nos estômagos analisados do T. lepturus dos quais se destacaramse: camarão, IND, teleósteos, Sardinella brasiliensis e sedimento. Essa diversidade esteve presente tanto no período de estiagem (julho/ dezembro), quanto no período chuvoso (janeiro/ junho), conforme a Tabela 2. 
Tabela 2 - Itens alimentares encontrados nos estômagos do Trichiurus lepturus capturados na costa maranhense.

\begin{tabular}{|c|c|c|c|c|c|c|c|c|c|}
\hline \multirow{2}{*}{$\begin{array}{l}\text { Itens } \\
\text { (presas) }\end{array}$} & \multicolumn{3}{|c|}{$\operatorname{Seco}(n=81)$} & \multicolumn{3}{|c|}{ Chuvoso $(n=39)$} & \multicolumn{3}{|c|}{ General $(n=120)$} \\
\hline & $\%$ Fni & $\% \mathrm{FO}$ & \%IRI & \%Fni & \%FO & \%IRI & \% Fni & $\% \mathrm{FO}$ & $\%$ IRI \\
\hline Camarão (peneídeos) & 1,56 & 2,47 & 1,00 & 4,00 & 5,13 & 2,66 & 2,23 & 3,33 & 1,47 \\
\hline IND & 31,00 & 48,15 & 22,53 & 20,00 & 25,64 & 14,92 & 27,93 & 40,83 & 20,41 \\
\hline Teleósteo & 51,17 & 43,21 & 58,67 & 60,00 & 56,41 & 66,34 & 53,64 & 47,50 & 60,79 \\
\hline $\begin{array}{l}\text { Sardinha (Sardinella } \\
\text { brasiliensis) }\end{array}$ & 16,27 & 6,17 & 17,80 & 14,00 & 10,26 & 13,50 & 15,64 & 7,51 & 16,61 \\
\hline Sedimento & 0,00 & 0,00 & 0,00 & 2,00 & 2,56 & 2,58 & 0,56 & 0,83 & 0,72 \\
\hline Total & 100 & 100 & 100 & 100 & 100 & 100 & 100 & 100 & 100 \\
\hline
\end{tabular}

Legenda: Fni- frequência numérica dos itens (\%Fni); FO- frequência de Ocorrência dos itens (\%FO); IIR- índice de importância relativa (\%IIR); IND-Item não identificado.

Nota: Devido ao grau de digestão, não foi possível caracterizar todos os teleósteos, sendo possível apenas a identificação da Sardinella.

Fonte: Autores (2021).

$\mathrm{Na}$ análise dos itens estomacais observou-se diferentes estágios de digestão, sendo estes: digerido (período de estiagem 52,17\% e no período chuvoso 48,07\%), parcialmente digerido (no período de estiagem 32,60\% e no período chuvoso $19,23 \%$ ), e semidigerido (no período de estiagem 15,21\% e no período chuvoso $32,69 \%$ ) respectivamente.

Os índices ecológicos dos itens alimentares do Trichiurus lepturus apresentaram os valores da diversidade com máxima de 1,71 bits cels $^{-1}$ em novembro/dezembro e mínimo de 0,77 bits cels $^{-1}$ nos meses de setembro /outubro. Com relação a riqueza, os valores foram máximos de 1,73 bits cels $^{-1}$ nos meses de março/abril e mínimo de 0,58 bits cels $^{-1} \mathrm{em}_{\text {julho/agosto. } \mathrm{O}}$ resultado da equitabilidade obtiveram uma máxima de 0,91 em março/abril e mínima de 0,70 nos meses de setembro /outubro (Figura 2).

Figura 2 - Frequência de Ocorrência dos itens alimentares em relação ao grau de digestão do T. lepturus capturados no litoral Maranhense.

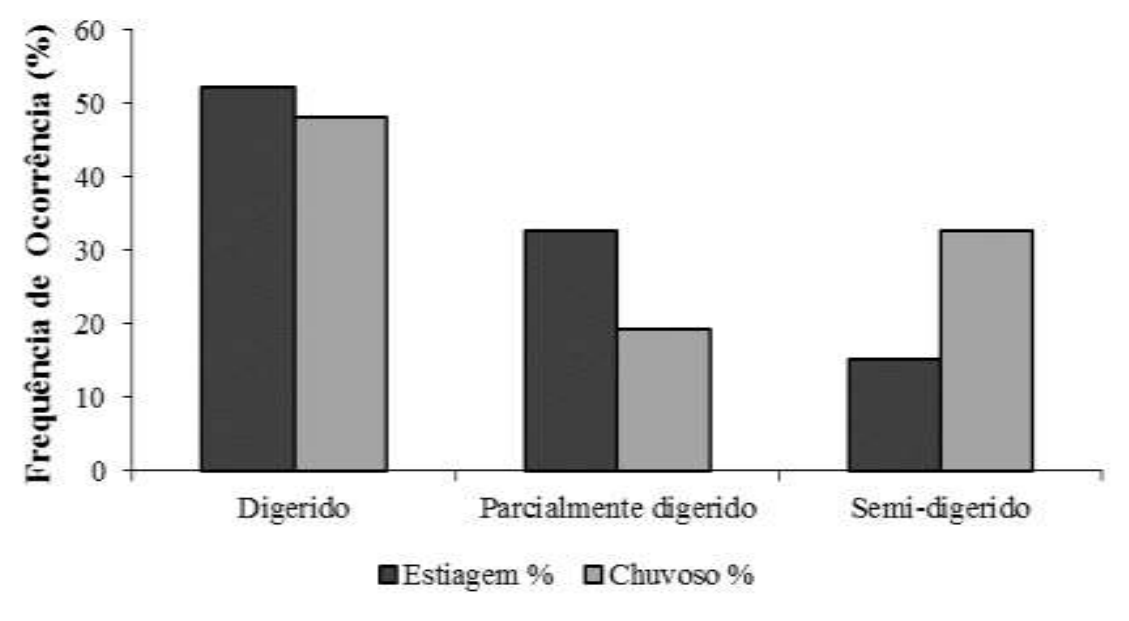

Fonte: Autores (2021). 
Quando se considera todos os itens alimentares, os teleósteos e IND foram os mais frequentes nos conteúdos estomacais dos adultos, ocorrendo em quase todas as amostras analisadas. Entre os juvenis, os alimentos mais comuns no trato digestivo foram IND e sedimento (Figura 3).

Figura 3 - Índices ecológicos dos itens alimentares do T. lepturus capturados na costa Maranhense.

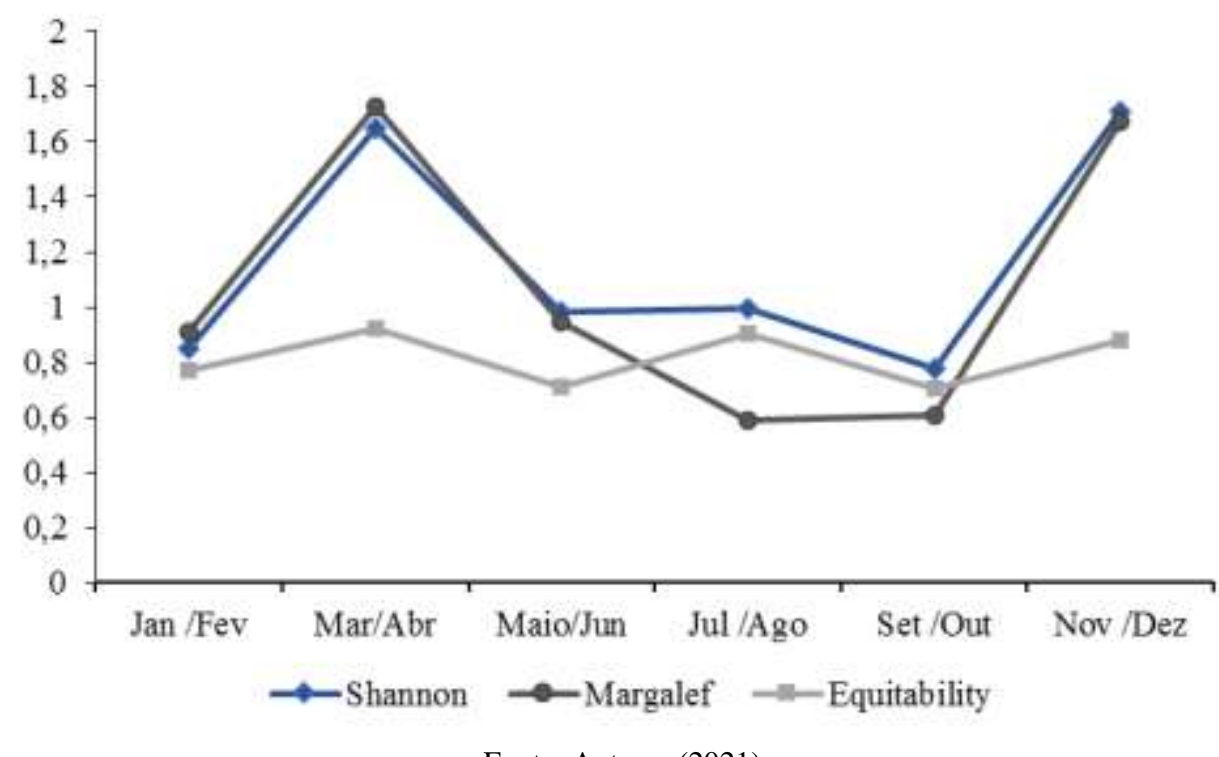

Fonte: Autores (2021).

Na Figura 4 podemos observar a frequência de ocorrência dos itens alimentares juvenis e adultos do T. lepturus, durante o período de estudo. Podemos constatar que ao longo de todas as amostras analisadas foram apresentados expressivos registros de IND na alimentação das fêmeas. Enquanto isso, os machos consumiram constantemente teleósteos, principalmente em julho/agosto e novembro/dezembro.

Figura 4 - Frequência de ocorrência dos itens alimentares do T. lepturus, capturados no litoral Maranhense no período de janeiro a dezembro de 2019.

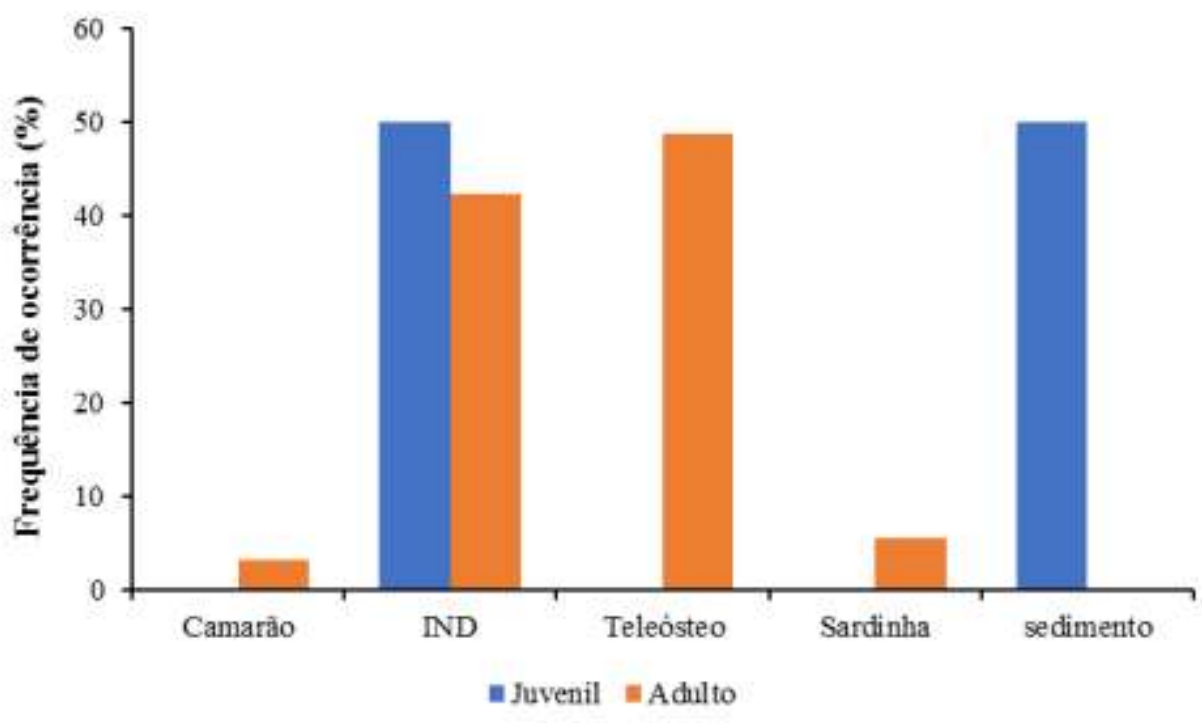

Fonte: Autores (2021). 
Os meses de julho a dezembro, registraram precipitação média de 34,43 $22,32 \mathrm{~mm}$, no qual corresponde ao período de estiagem, dessa forma constatou-se que esta espécie se alimentou em maior proporção, pois foi onde ocorreu o maior número de itens alimentares (Figura 5).

Figura 5 - Precipitação em relação a frequência de ocorrência bimestral dos itens alimentares de machos e fêmeas do $T$. lepturus capturados no litoral Maranhense, no período de janeiro a dezembro de 2019.

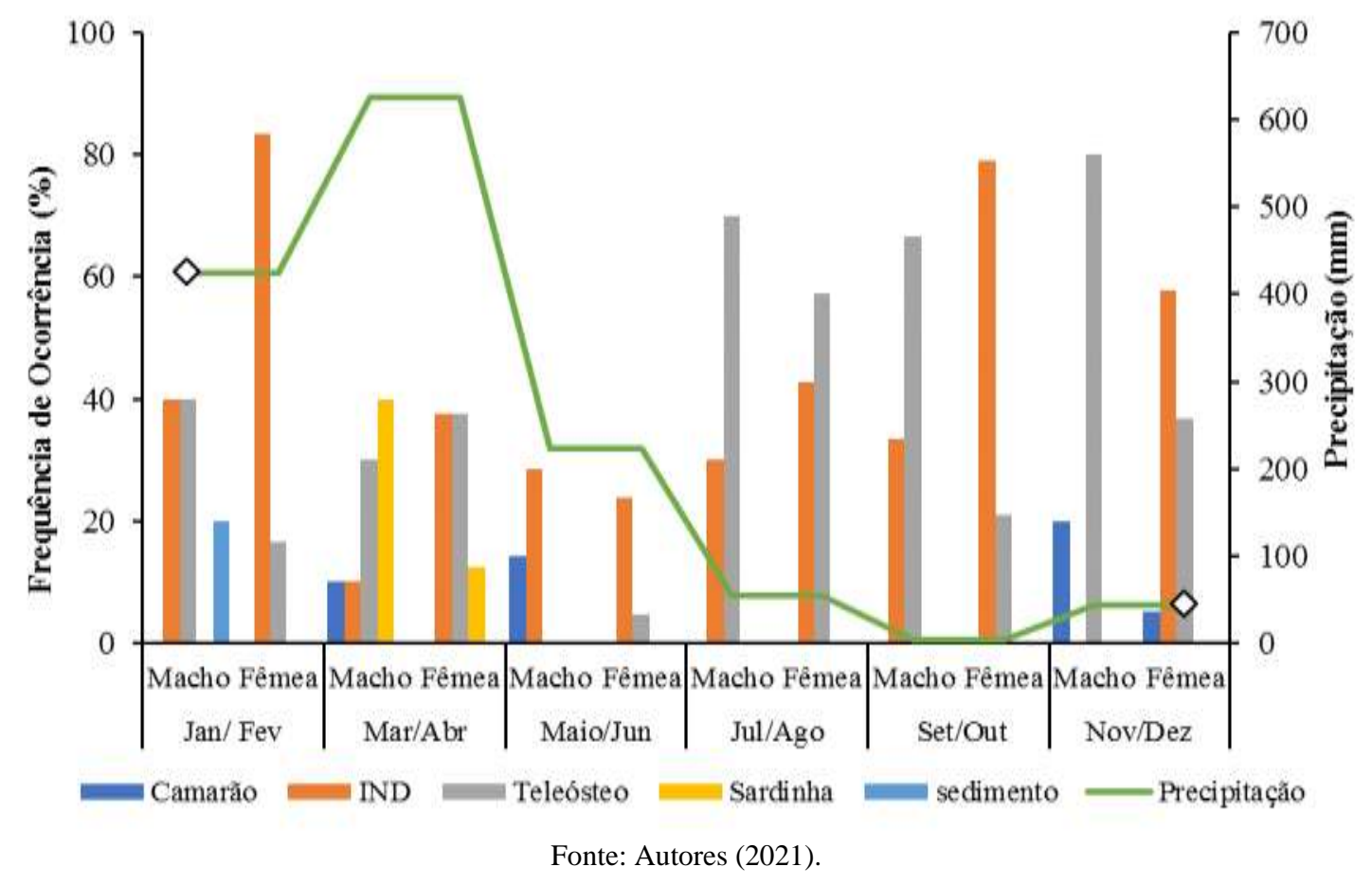

\section{Discussão}

A distribuição da T. lepturus no Brasil pode ser referente a sua adaptação aos ambientes que indicam grandes variações nas características físicas e biológicas, que pode ser explicada por sua adaptação para itens alimentares conjunta em diversos níveis tróficos e em uma heterogeneidade de reduzidas áreas espessas de presas, bem como, em áreas com presas maiores que são móveis em toda a coluna de água (Dou et al. 2000; Claessen \& Roos 2003; Martins et al. 2005; Fessehaye et al. 2006).

As análises dos conteúdos estomacais reforçaram a afinidade da espécie por alimentar-se de peixes, embora tenham sido encontrados peneídeos como complemento na alimentação da mesma. Isso corrobora com os resultados de Bittar et al. (2008), visto que no litoral do Rio de Janeiro, o hábito alimentar de T. lepturus fora predominantemente piscívoro, apesar do registro de crustáceos e cefalópodes, alimentando-se de presas pelágicas.

A espécie Sardinella brasiliensis, uma das principais presas do T. lepturus no presente estudo, distribui-se em faixa de profundidade entre 5 e $60 \mathrm{~m}$ (Gigliotti et al., 2010), o que pode ser indício de que a espécie em estudo apresenta movimentação ao longo do nível batimétrico na região.

A estrutura em comprimento de uma espécie ou população retrata as condições ambientais presentes e as precedentes nas quais as populações se manifestam (Agostinho, 1985). Em estudos realizados no Rio de Janeiro, os estômagos do $T$. lepturus possuíram uma média de $3 \mathrm{~cm}$ de comprimento (Bittar et al., 2008). O comprimento dos estômagos nesse estudo obteve média de 17,92 $\pm 5,6 \mathrm{~cm}$ no período chuvoso e média de $15,98 \pm 3,86 \mathrm{~cm}$ no período de estiagem. 
Estudo realizado por Winemiller (1989) apontou que peixes tropicais em ambientes sazonais como o T. lepturus geralmente podem ser adicionados aos estrategistas sazonais. Mateus et al. (2007) e Nunes et al. (2020), observaram que essas espécies aumentam seu estoque no período chuvoso, porque elas migram para a reprodução e desova total.

Obteve-se uma dada relevância na média dos itens alimentares como teleósteos, tendo assim, uma maior representatividade no período de estiagem com média de 51,17\%. Segundo Webb (1986), o estudo da morfologia funcional é capaz de apontar informações relevantes a respeito da ligação predador-presa e suas táticas. Quanto a separação da presa, Martins et.al (2005) e Bittar et. al (2008) julgam ser um comportamento normal da espécie fazê-lo em três pedaços.

A presença do T. lepturus, no litoral Maranhense pode ser explicada pelo fato de que a espécie demonstra possuir grande afinidade por alimentar-se de Sardinella brasiliensis espécie essa que desova em áreas costeiras (Matsuura 1998).

O hábito alimentar do T. lepturus consistiu, primordialmente, em uma dieta piscívora, embora tenha registros de itens alimentares como crustáceos, ou seja, predando espécies pelágicas, episódio que também foi registrado por Chiou et al. (2006) em Taiwan, e por Martins et al. (2005), Magro (2006) e Bittar (2008) no Brasil.

Como já mencionado na literatura (Chiou et al. 2006; Martins et al. 2005), no litoral maranhense foi observado a presença de resto de peixes, ou seja, os pedaços partidos explicam quanto a voracidade da espécie durante a sua alimentação facilitando a sua ingestão.

Na costa maranhense a dieta do T. lepturus variou sazonalmente. A intensidade dos principais itens consumidos diferenciou-se ao longo do ciclo hidrológico e apesar da IND ter uma participação importante na dieta da espécie no período de estiagem (julho/dezembro), houve uma diminuição no período chuvoso (janeiro/junho).

A precipitação não conseguiu influenciar na disponibilidade de alimento durante a pesquisa na costa maranhense, visto que foi constatado nos meses de março/abril de 2019 grande manifestação de chuvas, com precipitações elevadas de $625,95 \mathrm{~mm}$ a espécie não apresentou taxa elevada de estômagos cheios. Contudo, Junk et al. (1989), enfatiza que fortes chuvas podem afetar o balanço de nutrientes, a cadeia alimentar e ciclo de energia em uma determinada região. Assim corroborando com as afirmações de Junk (1985) e Santos (2010), sobre ocorrer maior taxa de alimentação das espécies no período de seca, do que no chuvoso.

\section{Conclusão}

A estacionalidade dos hábitos alimentares tem influência direta sobre a dinâmica populacional através de um sistema cíclico de predomínio trófico, em que os indivíduos se movimentam constantemente à procura da distribuição espacial ótima e maximize a sua função de predador. Esse conjunto de informações é fundamental para se obter dados que norteiam a adoção de estratégias e táticas sobre a participação do T. lepturus e outros recursos pesqueiros quanto a participação nos elos iniciais da cadeia trófica. Assim, a utilização pratica dessas informações servirá para otimização dos processos de gerenciamento da pesca, indicando o conhecimento da composição trófica e dos recursos pesqueiros específicos da área. A espécie apresentou hábito do tipo carnívoro, apesar de não possuir uma grande variação nos itens alimentares devido ao grau de digestão, a predominância foi por teleósteos e peneídeos, com maior influência alimentar no período de estiagem. É nítida a aptidão da espécie por peixes, bem como fora discorrido ao longo do trabalho, podendo assim concluir que embora outros itens sirvam de complemento alimentar, a Sardinella brasiliensis é o mais consumido por T. lepturus. 


\section{Agradecimentos}

Agradecemos ao apoio da Fundação de Amparo à Pesquisa e ao Desenvolvimento Científico e Tecnológico do Maranhão (FAPEMA), bem como, a Coordenação de Aperfeiçoamento de Pessoal de Nível Superior (CAPES), a Universidade Estadual do Maranhão (UEMA), por fim, ao Laboratório de Biologia Pesqueira (BIOPESQ).

\section{Referências}

Almeida, Z. S. (2009). Os recursos pesqueiros marinhos e estuarinos do Maranhão: Biologia, Tecnologia, Estado da Arte e Manejo. Tese de doutorado. Universidade Federal do Pará.

Andrade, T. C. et. al. (2011). Identificação do conteúdo estomacal de Peciformes e Carcharhiniformes: contribuição ao cruzeiro cientifico no sudeste e sul do Brasil (Dez/2009). Revista Ceciliana. 3(1), 45-49. http://sites.unisanta.br/revistaceciliana/edicao_05/1-2011-45 49.pdf

Agostinho, A. A. (1985). A estruturação, idade, crescimento e reprodução de Rhinelepis aspera (Agassiz, 1829) (Osteichthyes, Loricariidae) do rio Paranapanema, PR. 1985. 229p. Tese (Dutorado em Ciências Biológicas e Saúde), Centro de Ciências Biológicas e da Saúde, Universidade Federal de São Carlos.

Azevedo, A. C. G., Feitosa, F. A. N. \& Koening, M. L. (2008). Distribuição espacial e temporal da biomassa fitoplanctônica e variáveis ambientais no Golfão Maranhense, Brasil. Acta Botânica Brasilica, 22(3), 870-877. Doi: https://doi.org/10.1590/S0102-33062008000300022

Bittar, V. T. (2008). Hábito alimentar do peixe-espada adulto, Trichiurus lepturus, na costa norte do Rio de Janeiro, sudeste do Brasil. Revista Biotemas, Campos dos Goytacazes - RJ, Brasil, 83-90. http://www.uenf.br/Uenf/Downloads/pgecologia_5729_1217528732.pdf.

Cardoso, E. S. et. al. (2008). "Pesca e Atividade Complementares em Águas Interiores do Rio Grande do Sul". http://observatoriogeograficoamericalatina.org.mx/ega112/Procesosambientales/Usoderecursos/67.pdf.

Cervigón, F., R Cipriani, W. Fischer, L. Garibaldi, M. Hendrickx, A .J. Lemus, R. Márquez, J. M. Poutiers, Robaina B. \& Rodriguez, (1992). Fihas FAO de identificación de espécies para los fines de la pesca. Guía de campo de las espécies comerciales marinas y de aquas salobres de la costa septentrional de Sur América. FAO, Rome. 513p. Preparado con el financiamento de la Comisión de Comunidades Europeas y de NORAD.

Chiou, W. D., Chen, C. Y., Wang, C. M. \& Chen, C. T. (2006). Food and feeding habits of ribbonfish Trichiurus lepturus in coastal waters of south-western Taiwan. Fisheries Science, 72: 373-381.

Coelho, L. I. et. al. (2010). Contribuição ao conhecimento da dieta, atividade alimentar e reprodução de lolliguncula brevis (blainville, 1823) na região costeira de santos (estado de são paulo). bol. inst. pesca, são paulo, 3, 225-236”. http://www.pesca.sp.gov.br/36_3_2256rev.pdf

Claessen, D., Roos, A. M. (2003). Bistability in a size-structured population model of cannibalistic fish-a continuation study. Theoretical Population Biology, 64, 49-65.

Dou, S., Seikai, T. \& Tsukamoto, K. (2000). Cannibalism in japanese flounder juveniles, Paralichthys olivaceus, reared under controlled conditions. Aquaculture, 182, 149-159.

FAO. (2005) (Food and Agricultural Organization). http: //www.fao.org/figis/servlet/FiRefServet?ds=specie \&fid=2468

Fessehaye, Y., Kabir, A., Bovenhuis, H., \& Komen, H. (2006). Prediction of cannibalism in juvenile Oreochromis niloticus based on predator to prey weight ratio, and effects of age and stocking density. Aquaculture, 25 5: 314-322.

Fonteles-Filho, A.A., 2011. Oceanografia, biologia e dinâmica populacional de recursos pesqueiro. Expressão gráfica e Editora ed, p.464

Gigliotti, E. S. et. al. (2010). Spatial analysis of egg distribution and geographic changes in the spawning habitat of the brazilian sardine Sardinella brasiliensis. Journal of Fish Biology, 77(10): 2248-2267.

IBGE. (2012). https://www.ibge.gov.br/cidades-e-estados/ma/raposa.html.

Hyslop, E. J. (1980). Stomach contentes analysis; a review of methods and their application. Journal of Fish Biology, 17: 411-429.

Junk, W. J., Bayley, P. B. \& Sparks, R. E. (1989). The flood pulse concept in riverfloodplain systems. Canadian Journal of Fishers and Aquatic, 106: 110127.

Margalef, R. (1958). Temporal succession and spatial heterogeneity in phytoplankton. In: Buzzati-Traverso, A.A. (ed). Perspectives in marine biology. Berkeley: University of California. 323-349.

Magro, M. (2006). Aspectos da pesca e dinâmica de populações da espada, Trichiurus lepturus (Trichiuridae, Teleostei), da costa Sudeste-Sul do Brasil. Tese de Doutorado, Instituto Oceanografico, Universidade de São Paulo, Brasil,174pp.

Martins, A. S., Haimovici, M. (2000). Reproduction of the cutlassfish Trichiurus lepturus in the Southern Brazil subtropical convergence ecosystem. Scientia Marina, 64 (1): 97-105.

Martins, A. S., Haimovici, M. \& Palacios, R. (2005). Diet and feeding of the cutlassfish Trichiurus lepturus in the Subtropical Convergence Ecosystem of southern Brazil. Journal of Marine. Biology Ass. U.K. 85, 1223-1229.

Mateus, L. A. F., Penha, J. M. F. (2007). Dinâmica populacional de quatro espécies de grandes bagres na bacia do rio Cuiabá, Pantanal Norte, Brasil (Siluriformes, Pimelodidae). Rev. Bras. Zool. 24(1), 87-98. https://doi.org/10.1590/S0101-81752007000100012. 
Research, Society and Development, v. 10, n. 13, e81101320924, 2021

(CC BY 4.0) | ISSN 2525-3409 | DOI: http://dx.doi.org/10.33448/rsd-v10i13.20924

Matsuura, Y. (1998). Brazilian sardine (Sardinella brasiliensis) spawning in the southeast Brazilian Bight over the period 1976-1993. Revista Brasileira de Oceanografia, 46(1), 33-43.

Nunes, J. L. S., Piorski, N. M. (2011). Peixes marinhos e estuarinos do Maranhão. Café \& Lápis. p. 204

Nunes, Y. B. S. (2020). Estudo dos aspectos reprodutivos do trichiurus lepturus (linnaeus, 1758) na Costa Maranhense, Brasil. Brazilian Journal of Development, 6(1), 4201-4210. 10.34117/bjdv6n1-299.

Pielou, E. C. (1966). The measure of diversity in different types of biological collections. Journal of theoretical Biology, 13: 131-144.10.1016 / 0022-5193 (66) 90013-0.

Pinkas, L., Oliphant, M. S. \& Iverson, I. L. K. (1971). Food habits Food habits of albacore, bluefin tuna zand bonito in Californian waters. Fish Bulletin, 152: $1-105$.

Santos, R.N., Amadio, S. \& Ferreira, E.J.G. (2010). Patterns of energy allocation to reproduction in three Amazonian fish species. Neotropical Ichthyology, $8(1): 155-162$.

Shannon, C. E. W. (1963). The Mathematical Theory of Communication. Urbana: University Illinois Press; 117 p.

Vazzoler, A. E. A. M. (1996). Biologia da reprodução de peixes teleósteos: teoria e prática. Maringá, EDUEM, São Paulo. SBI, 169p.

Webb, P. W., Weihs, D. (1986). Functional locomotor morphology of early life history stages of fishes. Transactions of the American Fisheries Society, 115, 115-117.

Winemiller, K. O. (1989). Patterns of variation in life history among South American fishes in seasonal environments. Oecologia 81: 225-241. 